\title{
Microenvironment and its role in acquiring drug resistance of leukemia treatment
}

\author{
Malak Yahia Mohammed Qattan* \\ College of Applied Medical Sciences and Community Services, King Saud University, Saudi Arabia
}

\begin{abstract}
Bone marrow niche is the microenvironment where the leukemic cells residue and it support cell proliferation, metastasis and drug resistance by secretion of numerous factors including cytokines. When leukemic cells grow, they disrupt normal hematopoietic progenitor cell (HPC) bone marrow niches and create abnormal microenvironments. Also, niches recently have been linked to the development of drug resistance. One type of stem cell that resides in the bone marrow niche is called Mesenchymal stem cells (MSCs). Two microenvironment niches have been identified in BM; Osteoblastic and vascular, these niches provide the area in where the leukemia cells can escape from the chemotherapy-induced death and acquire drug resistance cells. Moreover, this review highlight briefly the role of exosomes in cell-to-cell communication and progression. The interaction between BM and leukemia cells through different proteins including cytokines, adhesion molecule, signal transduction pathways and hypoxia are mentioned simply.
\end{abstract}

MSCs have potential successful uses in regenerative medicine, but in this short review focusing on their potential role in supporting resistance in leukemia and the progress in elucidating the cellular components of HSC in BM.

\begin{abstract}
Abbreviations: HPC: Hematopoietic Progenitor Cell, MSCs: Mesenchymal Stem Cells, BM: Bone Marrow, ALL: Acute Lymphoblastic Leukaemia, WHO: World Health Organization, CXCL: CXCChemokine Ligand, Ang 1: Angiopoeitin 1,VLA-4: Very Late Antigen-4, SDF-1: Stromal Cells Express, MMP-9: Matrix Metalloproteinase-9 , GSK3: Glycogen Synthase $3 \beta$, HIF1 $\alpha$ : Hypoxia Inducible Factor $1 \alpha$
\end{abstract}

\section{Introduction}

Leukaemia refers to blood cancer and results from overproduction of leukocytes (white blood cells) in the bone marrow. Acute lymphoblastic leukaemia (ALL) is a malignant disorder of lymphoid progenitor cells [1]. The immature lymphocytes, commonly known as lymphoblasts or blast cells, undergo proliferation inside the bone marrow and prevent it from producing blood cells effectively.

Acute lymphoblastic leukemia (T-ALL) is derived from T lymphoid progenitors. T-ALL forms approximately $15 \%$ of ALL [2]. ALL occurs frequently in children under 15 years of age with peak prevalence occurring in the age group, 2-5 years [3]. ALL can also affect those between 15 and 25 years old, as well as elderly people. In 2010, 8.257 people in the UK were diagnosed with leukaemia [4]. Leukaemia is more common in males than in females [3].

There are two main types of ALL, namely, B-cell ALL and T-cell ALL. These types of ALL are only differentiated through immunophenotyping. The B-cell ALL is more common than the T-cell ALL. Most cases of B-cell ALL begin in the precursor B-cell. According to the World Health Organization (WHO), ALL may be classified using the cell line as B-cell and T-cell and also using the cell maturity as precursor or mature.

Based on WHO's classification, ALL has 4 further subtypes which are Precursor B-cell ALL, Transitional pre-B ALL, B-cell ALL and T-cell ALL. Precursor B-Type cell ALL has a higher prevalence as it accounts for $80-85 \%$ of all acute lymphoblastic leukaemia cases.
Transitional pre-B ALL is characterized by unclear clinical picture and rare occurrence. It accounts for only $3 \%$ of all acute lymphoblastic leukaemia cases. B-cell ALL accounts for only 2-3\% while T-cell ALL affects up to $13 \%$ of all acute lymphoblastic leukaemia cases.

Niches are local tissue microenvironment to maintain and regulate stem cells maintenance, production and differentiation [5]. The stem cell niche concept was first introduced in 1970 by Schofield [6]. Bone marrow niches support leukemic cell proliferation, metastasis and drug resistance by secretion of numerous factors including cytokines. When leukemic cells grow, they disrupt normal hematopoietic progenitor cell (HPC) bone marrow niches and create abnormal microenvironments. Also, niches have been linked to the development of drug resistance $[7,8]$.

One type of stem cell that resides in the bone marrow niche is called Mesenchymal stem cells (MSCs) [9]. MSCs are adherent, non haematopoietic stem cells and have the ability to self-renew and to differentiate into multiple lineages including osteoblasts, chondrocytes and adipocytes [10]. MSCs express markers such as CD29, CD44, CD51, CD73 (SH3/4), CD105 (SH2), CD166 (ALCAM) and Stro-1. MSCs express a wide range of molecules, including growth factors, chemokines, adhesion molecules and toll-like receptors, on their surface. In terms of cell migration, MSCs are known to functionally

Correspondence to: Malak Yahia Mohd. Qattan, Assistant professor in Stem Cells and Leukemia Research, Vice director of Health Science Department, College of Applied Medical Sciences and Community Services, King Saud University, Riyadh, Saudi Arabia, Tel: 00966556333337/00966-118057228, E-mail: mqattan@KSU.EDU.SA

Key words: mesenchymal stem cells, bone marrow niche, microenvironment

Received: December 11, 2016; Accepted: December 28, 2016; Published: December 30, 2016 
express chemokine receptors CCR1, CCR4, CCR7, CCR9, CCR10, CXCR4, CXCR5, CXCR6, CX3CR1 and c-me [10]. In the bone marrow, there are two types of supportive niches for HSC, namely, the Endosteum niche (Osteoblastic) and the vascular niche [11] which are discussed later.

Understanding the factors involved in secreted factors' effect on leukemia is essential to ultimately develop novel clinical strategies aimed at using MSCs as vehicles to deliver antitumor factors or block actions of secreted factors to reduce tumor growth. The potential role of MSCs in tumor initiation or promotion is a significant concern that must be addressed fully to allow MSC-mediated therapy for cancer to realize its full potential [12].

\section{Endosteal niche and HSC maintenance}

Endosteum is highly vascularized interface between the bone and the bone marrow [13]. Endosteum is covered by a layer of bone cells that give rise to bone forming cells called osteoblasts. Besides osteoblasts, endosteum contains osteoclasts which are bone resorbing cells. Osteoblasts and osteoclasts play a crucial role in bone formation and remodeling. Both cells are found in endosteum in the equilibrium state [13].

Osteoblasts secrete factors to streamline the HSC in the bone marrow so as to maintain bone marrow quiescence. These positive factors include angiopoeitin 1 (Ang 1), thrombopoietin and CXC-chemokine ligand 12 (CXCL12). Angiopoeitin 1 (Ang 1) and thrombopoietin are responsible for controlling HSC quiescence, whereas, CXC-chemokine ligand 12 (CXCL12) is responsible for regulating HSC movement and stability in the bone marrow [13].

HSC obtain osteoblasts factors using different means such as direct adhesion to the osteoblasts, or by communication with neighboring cells and releasing of soluble factors when $\mathrm{N}$-cadherin ligand is not vital [13]. Osteoclasts express matrix metalloproteinase 9 (MMP9), cathepsin $\mathrm{K}$ and CXCL12 to contribute in HSC maintenance and localization inside the bone marrow.

\section{Vascular niche and HSC maintenance}

The sinusoidal niche is vascularized with a large number of sinusoids in haematopoeitic tissues. The sinusoids are small blood vessels in haematopoeitic tissue that allows cells trafficking in and outside the bone marrow [13]. The interface between the bone cell, the HSC and the endothelial cells are crucial for haemopoeisis (formation of blood cells) and bone formation.

Sinusoids are lined by endothelial cells which allow hematopoietic cells to go in and out of the circulation. The presence of $60 \%$ of HSC around the sinusoids reflects vascular function in maintaining HSC. Twenty percent of the cells are found inside the endosteum and the rest are distributed in the bone marrow [13]. Thus, the HSC microenvironment quiescence is maintained by osteoblastic niches, while both the proliferation and differentiation of the cells are supported by vascular niche [6]. Vascular niche plays three important roles in the bone marrow; It supplies the nutrients, oxygen and growth factors for the HSC cells for proliferation and differentiation. Sinusoidal endothelium along with chemokines encourages homing and mobilization of HSC. This occurs through endothelial mobilization when the HSC enters the vascular system, and homing when cell returns back to the bone marrow. A vascular niche is important for cells outside the bone marrow, such as in the spleen which may replace $\mathrm{BM}$ niche in case of bone marrow suppression [14].

\section{Signalling pathway in the bone marrow niche}

\section{Chemokine involvement in HSC homing and mobilization}

Chemokines are involved in HSC homing and mobilization. The ability of HSCs to mobilize and then return, or home, to the niche is regulated by specific molecules called chemokines. Chemokines are proteins secreted by the cell. The chemokine SDF-1 and its receptor CXCR4 play a crucial role in HSC mobilization and homing regulation. Endothelial cells, osteoblasts, and other stromal cells express SDF1, while HSCs express CXCR4. Moreover, activation of adhesion molecules such as the very late antigen-4 (VLA-4) and the leukocyte function antigen-1 (LFA-1) is also required for this process and also for the subsequent migration of $\mathrm{BM}$ toward the osteoblast surface. Likewise, high levels of SDF-1 on the surface of osteoblasts attract HSCs to return home to the osteoblast niche. The Rho GTPases Rac1 and Rac2, in response to SDF-1 signals, are also involved in regulating HSC mobilization and homing [15].

Granulocyte colony-stimulating factor (G-CSF) inactivates SDF1 in the bone marrow using proteolytic enzymes such as elastase, cathepsin G, matrix metalloproteinase-2 (MMP-2) and matrix metalloproteinase-9 (MMP-9) [14]. Homing of HSC to the osteoblast niche occurs when osteoblasts surface demonstrates raised level of SDF-1. HSC cells that express CXCR4, directly attach to SDF-1 on the surface of osteoblastic niches. Adhesion is strengthened by the $\mathrm{N}$-cadherin and $\beta$-catenin complex [15].

\section{Notch signaling}

Notch proteins are transmembrane receptors consisting of an extracellular amino-terminal and an intracellular carboxy-terminal subunits that are non-covalently linked by the heterodimerization domain (HD)[16]. Notch signaling has been found active in the BM niche as it regulates HSC self-renewal, quiescence and programmed cell death (apoptosis) [17]. As Notch signaling is very complexes signaling, limited information is available regarding the interaction of the signaling in the BM niche and the leukemia cells.

In T-ALL cells, Notch 3 signaling is highly expressed from the blood vessels endothelial cells in the BM microenvironment, Notch3 together with DLL-4 can promote tumor escape from the quiescence [18]. Moreover, Notch 3 silencing affected T-ALL cell survival, which indicates the mechanism of vascular niche in tumor progression [18].

\section{WNT/ $\beta$-catenin signaling}

The WNT signaling pathways are a group of signal transduction pathways made of proteins that pass signals into a cell through cell surface receptors. Three WNT signaling pathways have been characterized: the canonical WNT pathway, the noncanonical planar cell polarity pathway (WNT-PCP), and the noncanonical Wnt/calcium pathway $\left(\mathrm{WNT}-\mathrm{Ca}{ }^{2+}\right)$. All three pathways are activated by binding a WNT -protein ligand to a Frizzled family receptor, thus allow $\beta$-catenin to passes translocate to the nucleus, bind transcriptional factor and control target gene transcription. WNT/ $\beta$-catenin signaling pathway regulates variety of development function in different tissue including the hematopoietic tissue [19]. In leukemia, WNT signaling is expressed in B-cell and BM-MSCs in higher level than the normal status [20]. Activated WNT by BM microenvironment protects B-ALL from death to chemotherapy [20]. Furthermore, B-ALL cells express increase phosphorylation which inhibits glycogen synthase 3 (GSK3) $\beta$ which acts as $\beta$-catenin inhibitor [8]. Treatment with $\beta$-catenin inhibitor (XAV939) sensitized leukemia cells to therapy [21]. 


\section{Hypoxia}

Low Oxygen level is essential to maintain healthy BM microenvironment function [22]. The hypoxic tumors are found to be more resistance to chemotherapy. The most known marker of hypoxia is Hypoxia inducible factor $1 \alpha$ (HIF1a) [8].

Activation of transcription factor (HIFla) activates a variety of genes that play a role in metabolism, angiogenesis, apoptosis, and cell cycle progression [23]. Moreover, (HIF1a) is overexpressed in ALL patients and is considered as bad prognosis indicator [23]. However, (HIF1a) up-regulates WNT signaling pathway under hypoxic condition in T-ALL, leading to WNT/ $\beta$-catenin and HIFla support T-ALL cells [24]. Different strategies are developing targeting HIF1 $\alpha$ to exert hypoxia pro-survival effects of neoplastic cells [8].

\section{Adhesion molecules}

Adhesion molecules including osteopontin, VLA-4/VAM-1, LFA1/intercellular adhesion molecule-1 (ICAM-1), and N-cadherin are involved in $\mathrm{BM}$ homeostasis. The Adhesion molecules regulate the binding of HSCs to and from BM niche for mobilization, homing as well as providing signals for proliferation of HSC and quiescence [15].

High expression of VLA-4 is considering as a poor prognosis indicator in B-ALL patients [25]. Moreover, VLA-4 corresponds with cancer high risk patients group, suggesting that VLA-4 expression is decisive for leukemia cell survival and therefore affect therapy respond [25].

\section{Cytokines}

Cytokines including IL-7, IL-10, IL-15 and IFN- $\gamma$ together with their receptors are highly expressed in BM samples of B-ALL relapsed patients [26]. As well, cytokines is highly expressed from stromal cells from leukemic BM [27]. In in vitro studies on ALL cells, IL-7 cytokine was progression factor [28]. BM-MSCs express IL-7 which which prolong proliferation and survival of T-ALL [29]. Moreover, another cytokine was significantly increased which is IL-8. IL-8 can increase cell adhesion partially but not survival in leukemia cell, which highlight the role of IL-8 in establishment of malignant microenvironment [30]

\section{Exsosomes and microvesical}

Extracellular vesicles (EV) are released from the healthy or cancer cell under stress. The EV transfer signaling molecules such as microRNAs (miRNA) in functional active form. The EV are classified according to their size into Exosomes, Ectosome and apoptotic bodies. Exsosome is the smallest in size as the diameter varied from $40-150 \mathrm{~nm})$, Ectosome is larger in size (50-1000nm) and formed from plasma membrane, and apoptotic bodies that produced from blebbing membrane formed from cells undergo programmed cell death.

Exosomes carry a variety of proteins including MHC molecules, chaperones, receptors, receptors ligand and cytokines. Once exosome is produced from stressed cells, it binds to the recipient cell receptors to export the proteins and translated into functional protein. Notably, the exosome content in healthy and neoplastic cells are completely different which indicates the role of exosomes in development and progression of as well as chemo resistance and metastasis. Tumor cell exosome is released from neoplastic cells to allow permissive and tumour promoting microenvironment and it has been found in AML patients and melanoma model $[31,32]$. Multiple studies indicate that, the exosomes derived from tumor play a crucial role in metastasis and progression of cancer cells $[33,32]$

\section{Conclusion and therapy target}

Recent studies highlighted the role of bone marrow stem cells in developing resistance and failure of the treatment and subsequently death of the patient. This review has discussed several mechanisms behind $\mathrm{BM}$ microenvironment drug resistance. It is still not clearly understood how leukemic cells bring about modifications in the microenvironment [11]. During leukemia, number of CD34+ cells reduces with time and they become unable to travel to the peripheral circulation under the influence of cytokine stimulation. Neutralization of stem cell factor (SCF) released by leukemic cells blocks transport of CD34+ cells to malignant niches, normalizes the number of CD34+ cells and restores movement of CD34+ cells. HPC dysfunction is caused by tumour microenvironment through disrupting the normal HPC niches. However, the normal progenitor cell function can be sustained in malignant setting by the help of therapeutic blockage of HPC interaction with tumour niches [11]. In addition, CXCR4 is expressed on the surface of leukemic cells in order to facilitate leukemic cells homing in the BM microenvironment, followed by targeting additional leukemic cells in the microenvironment of bone marrow [14].

In addition, the presence of stromal cell derived exosomes in the bone marrow derived CM which produces soluble factors that induce a redox adaptation in ALL cells leading to chemoresistance. Moreover, exosomes that affect ALL cells response to drugs contain several micro RNA's. That may act through PI3K pathway.

Understanding the factors involved in secreted factors' effect on leukemia is essential to ultimately develop novel clinical strategies aimed at using MSCs as vehicles to deliver antitumor factors or block actions of secreted factors to reduce tumor growth. The potential role of MSCs in tumour initiation or promotion is a significant concern that must be addressed fully to allow MSC-mediated therapy for cancer to realize its full potential.

\section{References}

1. Yeoh EJ, Ross ME, Shurtleff SA, Williams WK, Patel D, et al. (2002) Classification, subtype discovery, and prediction of outcomein pediatric acute lymphoblastic leukemia by gene expression profiling. Cancer cell 1: 133-143. [Crossref]

2. Cardoso BA, Gírio A, Henriques C, Martins LR, Santos C, et al. (2008) Aberrant signaling in T-cell acute lymphoblastic leukemia: biological and therapeutic implications. Braz J Med Biol Res 41: 344-350. [Crossref]

3. Pui CH, Evans WE, Relling MV (2008) Are children with lesser-risk B-lineage acute lymphoblastic leukemia curable with antimetabolite therapy? Nat Clin Pract Oncol 5: 130-131. [Crossref]

4. (2013) Cancer Research UK, U. C. I.

5. Morrison SJ, Scadden DT (2014) The bone marrow niche for haematopoietic stem cells. Nature 505: 327-334. [Crossref]

6. Arai F, Hirao A, Suda T (2005) Regulation of hematopoietic stem cells by the niche. Trends Cardiovasc Med 15: 75-79. [Crossref]

7. Bakker E, Qattan M, Mutti L, Demonacos C, Krstic-Demonacos M (2015) The role of microenvironment and immunity in drug response in leukemia. Biochim Biophys Acta 1863: 414-426. [Crossref]

8. Chiarini F, Lonetti A, Evangelisti C, Buontempo F, Orsini E, et al. (2016) Advances in understanding the acute lymphoblastic leukemia bone marrow microenvironment From biology to therapeutic targeting. Biochim Biophys Acta 1863: 449-463. [Crossref]

9. Herzog EL, Chai L, Krause DS (2003) Plasticity of marrow-derived stem cells. Blood 102: 3483-3493. [Crossref]

10. Dwyer RM, Khan S, Barry FP, O'Brien T, Kerin MJ (2010) Advances in mesenchymal stem cell-mediated gene therapy for cancer. Stem Cell Res Ther 1: 25. [Crossref]

11. Colmone A1, Amorim M, Pontier AL, Wang S, Jablonski E, et al. (2008) Leukemic Cells Create Bone Marrow Niches That Disrupt the Behavior of Normal Hematopoietic Progenitor Cells. Science 322: 1861-1865. [Crossref] 
12. Spaeth E, Klopp A, Dembinski J, Andreeff M, Marini F (2008) Inflammation and tumor microenvironments: defining the migratory itinerary of mesenchymal stem cells. Gene Ther 15: 730-738. [Crossref]

13. Kiel MJ, Morrison SJ (2008) Uncertainty in the niches that maintain haematopoietic stem cells. Nat Rev Immunol 8: 290-301. [Crossref]

14. Yin T, Li L (2006) The stem cell niches in bone. J Clin Invest 116: 1195-1201. [Crossref]

15. Kopp HG, Avecilla ST, Hooper AT, Rafii S (2005) The bone marrow vascular niche: home of HSC differentiation and mobilization. Physiology (Bethesda) 20: 349-356. [Crossref]

16. Zhao D, Xue C, Lin S1, Shi S1, Li Q1, et al. (2016) Notch Signaling Pathway Regulates Angiogenesis via Endothelial Cell in 3D Co-Culture Model. J Cell Physiol. [Crossref]

17. Azizidoost S, Bavarsad MS, Bavarsad MS, Shahrabi S, Jaseb K, et al. (2015) The role of notch signaling in bone marrow niche. Hematology 20: 93-103. [Crossref]

18. Indraccolo S, Minuzzo S, Masiero M, Pusceddu I, Persano L, et al. (2009) Cross-talk between tumor and endothelial cells involving the Notch3-D114 interaction marks escape from tumor dormancy. Cancer Res 69: 1314-1323. [Crossref]

19. Malhotra S, Kincade PW (2009) Wnt-related molecules and signaling pathway equilibrium in hematopoiesis. Cell Stem Cell 4: 27-36. [Crossref]

20. Khan NI, Bradstock KF, Bendall LJ (2007) Activation of Wnt/beta-catenin pathway mediates growth and survival in B-cell progenitor acute lymphoblastic leukaemia. $\mathrm{BrJ}$ Haematol 138: 338-348. [Crossref]

21. Hu K, Gu Y, Lou L, Liu L, Hu Y, et al. (2015) Galectin-3 mediates bone marrow microenvironment-induced drug resistance in acute leukemia cells via Wnt/B-catenin signaling pathway. J Hematol Oncol 27: 1. [Crossref]

22. Benito J, Zeng Z, Konopleva M, Wilson WR (2013) Targeting hypoxia in the leukemia microenvironment. Int J Hematol Oncol 2: 279-288. [Crossref]

23. Benito J, Shi Y, Szymanska B, Carol H, Boehm I, et al. (2011) Pronounced hypoxia in models of murine and human leukemia: high efficacy of hypoxia-activated prodrug PR-104. PLoS One 6: e23108. [Crossref]

24. Giambra V, Jenkins CE, Lam SH, Hoofd C, Belmonte M, et al. (2015) Leukemia stem cells in T-ALL require active Hifl $\alpha$ and Wnt signaling. Blood 125: 3917-3927. [Crossref]

25. Shalapour S, Hof J, Kirschner-Schwabe R, Bastian L, Eckert C, et al. (2011) High VLA-4 expression is associated with adverse outcome and distinct gene expression changes in childhood B-cell precursor acute lymphoblastic leukemia at first relapse. Haematologica 96: 1627-1635. [Crossref]

26. Kebelmann-Betzing C, Körner G, Badiali L, Buchwald D, Möricke A, et al. (2001) Characterization of cytokine, growth factor receptor, costimulatory and adhesion molecule expression patterns of bone marrow blasts in relapsed childhood B cell precursor all. Cytokine 13: 39-50. [Crossref]

27. Wu S, Korte A, Kebelmann-Betzing C, Gessner R, Henze G, et al. (2005) Interaction of bone marrow stromal cells with lymphoblasts and effects of predinsolone on cytokine expression. Leuk Res 29: 63-72. [Crossref]

28. Barata JT, Cardoso AA, Boussiotis VA(2005) Interleukin-7 in T-cell acute lymphoblastic leukemia: an extrinsic factor supporting leukemogenesis? Leuk Lymphoma 46: 483495. [Crossref]

29. Scupoli MT, Perbellini O, Krampera M, Vinante F, Cioffi F, et al. (2007) Interleukin 7 requirement for survival of T-cell acute lymphoblastic leukemia and human thymocytes on bone marrow stroma. Haematologica 92: 264-266. [Crossref]

30. de Vasconcellos JF, Laranjeira AB, Zanchin NI, Otubo R, Vaz TH, et al. (2011) Increased CCL2 and IL- 8 in the bone marrow microenvironment in acute lymphoblastic leukemia. Pediatr Blood Cancer 56: 568-577. [Crossref]

31. Boyiadzis M, Whiteside TL (2015) Information transfer by exosomes: A new frontier in hematologic malignancies. Blood Rev 29: 281-290. [Crossref]

32. Peinado H, Alečković M, Lavotshkin S, Matei I, Costa-Silva B, et al. (2012) Melanoma exosomes educate bone marrow progenitor cells toward a pro-metastatic phenotype through MET. Nat Med 18: 883-891. [Crossref]

33. Vallabhaneni KC, Penfornis P, Dhule S, Guillonneau F, Adams KV, et al. (2015) Extracellular vesicles from bone marrow mesenchymal stem/stromal cells transport tumor regulatory microRNA, proteins, and metabolites. Oncotarget 6: 4953-4967. [Crossref]

Copyright: (C2016 Qattan MYM. This is an open-access article distributed under the terms of the Creative Commons Attribution License, which permits unrestricted use, distribution, and reproduction in any medium, provided the original author and source are credited. 